\title{
CONTROL POST LEGISLATIVO EN LOS PARLAMENTOS: EL CASO DE CHILE Y EL REINO UNIDO
}

\begin{abstract}
Constanza TORO*
"Post legislative scrutiny appears to be similar to motherhood and apple pie in that everyone appears to be in favour of it. However, unlike motherhood and apple

Resumen

Este artículo desarrolla un estudio comparativo entre Chile y el Reino Unido en relación con el funcionamiento del sistema de escrutinio post legislativo. Para evaluarlo, se hace una referencia breve a los distintos tipos de escrutinio legislativo, para luego enfocarnos en el escrutinio que llevan a cabo los Parlamentos una vez que se promulga una ley. La comparación entre ambas naciones se realiza de acuerdo con el cumplimiento de los siguientes estándares: (i) Efectividad, (ii) Eficacia y (iii) Eficiencia. Este artículo examina la forma como los sistemas de escrutinio post legislativo en Chile y el Reino Unido alcanzan cada uno de los objetivos mencionados, concluyendo que, en términos generales, el modelo del Reino Unido representa un sistema de escrutinio post legislativo más exitoso. No obstante, se proponen algunas recomendaciones para mejorar los sistemas en ambos países.
\end{abstract} pie, it is not much in evidence". 1

Palabras Clave: Chile, Reino Unido, Parlamento, escrutinio post legislativo, evaluación de la legislación.

\section{INTRODUGGIÓN}

El objetivo último de la legislación es ser aplicada y lograr los resultados perseguidos al momento de ser propuesta y promulgada. Son muchos los factores que pueden influir en este proceso, desde el diseño de las políticas al proceso de redacción, incluyendo la implementación. Del mismo modo, son muchos los actores que juegan un rol en este proceso, incluyendo al gobierno, la legislatura, los tribunales,

* Comisión de Constitución, Legislación y Justicia de la Cámara de Diputados, Valparaíso, Chile (constanza.toro@gmail.com). Artículo recibido el 12 de octubre de 2017, aceptado para su publicación el 27 de febrero de 2018. Traducción de Daniela Pavez.

1 House of Lords (2004), Par. 165: "La evaluación legislativa ex post se parece a la maternidad y al pastel de manzana en que todos están a favor de ella. Sin embargo, a diferencia de la maternidad y el pastel de manzana, hay pocas evidencias sobre ello". 
etc. En este artículo me enfocaré en un aspecto específico, vinculado con lograr una legislación exitosa: el escrutinio post legislativo realizado por los parlamentos.

Para examinar este aspecto, estudiaré los casos de Chile y el Reino Unido, ofreciendo un análisis comparado que dará luz sobre ciertos componentes del escrutinio post legislativo. Al estudiar ambos modelos utilizaremos como criterio comparativo los estándares establecidos por Luzius Mader para evaluar la legislación: (i) efectividad, (ii) eficacia y (iii) eficiencia. ${ }^{2}$ Estos criterios fueron elegidos porque se consideran un marco de análisis comprehensivo, que permite considerar la evaluación de la legislación desde un punto de vista técnico, pero también considerando su desempeño como herramienta de política pública. La hipótesis planteada en este artículo es que el modelo de escrutinio parlamentario post legislativo del Reino Unido cumple mejor con los criterios expuestos por Mader, aun cuando dicho modelo funciona como un sistema de control difuso.

La metodología seguida en este artículo es la siguiente: primero, presentaré el tópico del escrutinio legislativo, los diferentes tipos de controles existentes y cómo ellos interactúan entre sí. Luego, explicaré los criterios de Mader y el significado de los tres estándares que guiarán este análisis. Posteriormente, desarrollaré un análisis comparativo examinando los modelos de escrutinio post legislativo parlamentario tanto en Chile como en el Reino Unido, señalando cómo ellos cumplen con cada uno de los estándares. Además, se presentará un caso de estudio para ilustrar cómo ambos Parlamentos evaluaron la legislación en materia de salud mental. Para finalizar, presentaré algunas conclusiones, destacando algunas recomendaciones que pueden ayudar a fortalecer ambos modelos.

\section{ASPEGTOS GENERALES DEL ESGRUTINIO LEGISLATIVO}

Existen múltiples formas de evaluar la política legislativa. Por lo mismo, es necesario comenzar introduciendo ciertas clarificaciones conceptuales, con el fin de delimitar el área específica abarcada por el escrutinio parlamentario post legislativo. La primera distinción se refiere a quien realiza la evaluación: si es el gobierno, el parlamento, una agencia independiente, una comisión de expertos o algún otro actor posible. Una segunda distinción se refiere al momento en el cual se realiza la evaluación, reconociendo que la evaluación puede llevarse a cabo antes de que la política entre en vigor, conocida como evaluación ex ante, pre-legislativa, o prospectiva; o bien, puede ser realizada una vez que la política se encuentra en vigor, conocida como evaluación ex post, post legislativa, o retrospectiva. ${ }^{3}$

El Departamento de Empresas, Innovación y Formación (BIS) se refiere al escrutinio post legislativo (PLS, por sus siglas en inglés) como uno de los tres tipos de revisión ex post existentes, junto con la política de evaluación y la revisión post

2 Mader (2001), p. 119.

3 Algunos autores agregan una tercera instancia de evaluación, realizada durante el proceso legislativo. KarPen (2004), p. 310. 
implementación (PIR, por sus siglas en inglés). Sin embargo, aun cuando la revisión post implementación y el escrutinio post legislativo son formas de evaluar una política, ellas no son sinónimas. En ese sentido, la política de evaluación es el término más genérico para referirse a una evaluación sistemática de una política regulatoria. El PIR, en cambio, tiene por objetivo ser un complemento de la evaluación ex ante, realizada en el contexto de la "evaluación de impactos". En consecuencia, la PIR es una "versión revisada" de la evaluación de impactos, mientras que la PLS tiene por finalidad revisar cómo funciona la legislación en la práctica.

De acuerdo con lo señalado por el BIS, en el escrutinio post legislativo:

la audiencia primaria es el Parlamento; lo que incluye una revisión para determinar en qué medida la legislación y la legislación secundaria que la apoya, han entrado en vigor. A diferencia del PIR, esto incluye una revisión sobre hasta qué punto la legislación y la legislación secundaria que le da fundamento han entrado en vigor. Así, el escrutinio post legislativo debiera considerar todo o gran parte de la legislación delegada elaborada en virtud de una ley ["primary audience is Parliament, it includes a review of the extent to which the legislation and the supporting secondary legislation has been brought into force. Unlike PIR, it includes a review of the extent to which the legislation and the supporting secondary legislation has been brought into force. Post legislative scrutiny should include consideration of all or much of the delegated legislation made under the Act']. ${ }^{4}$

El siguiente gráfico explica esta relación:

Evaluación de las Políticas

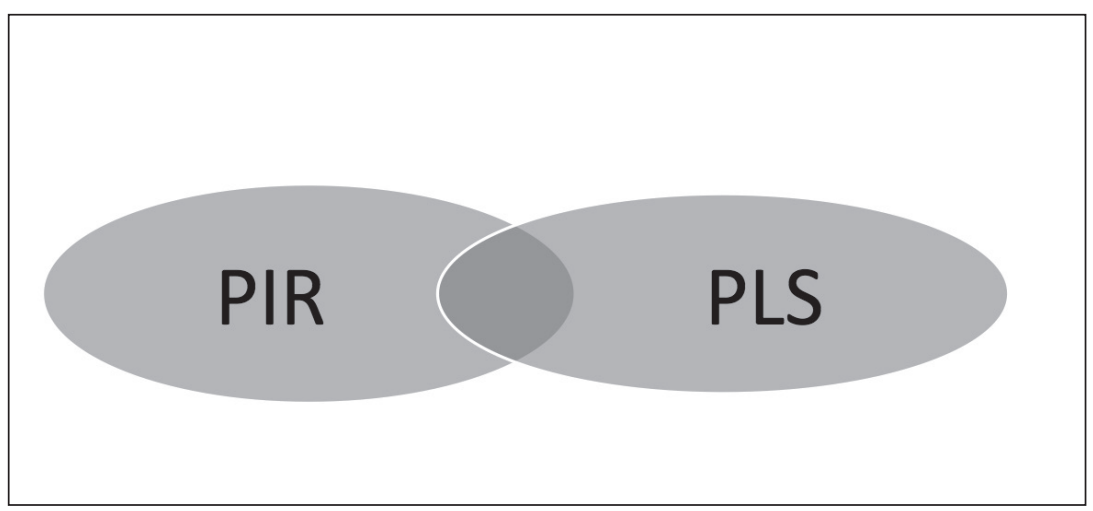

Existe una relación estrecha entre las diferentes categorías de escrutinio legislativo. Por un lado, la evaluación realizada por los Parlamentos se encuentra estrechamente conectada con la evaluación del gobierno y otras agencias; por otro lado, la evaluación ex post debe ser entendida en relación con la evaluación ex ante. Esta última relación ha sido explicada por la OCDE en el siguiente esquema:

$4 \quad$ BIS (2010), p. 2. 


\section{Gráfico 2. Etapas del ciclo de las político-regulatorio}

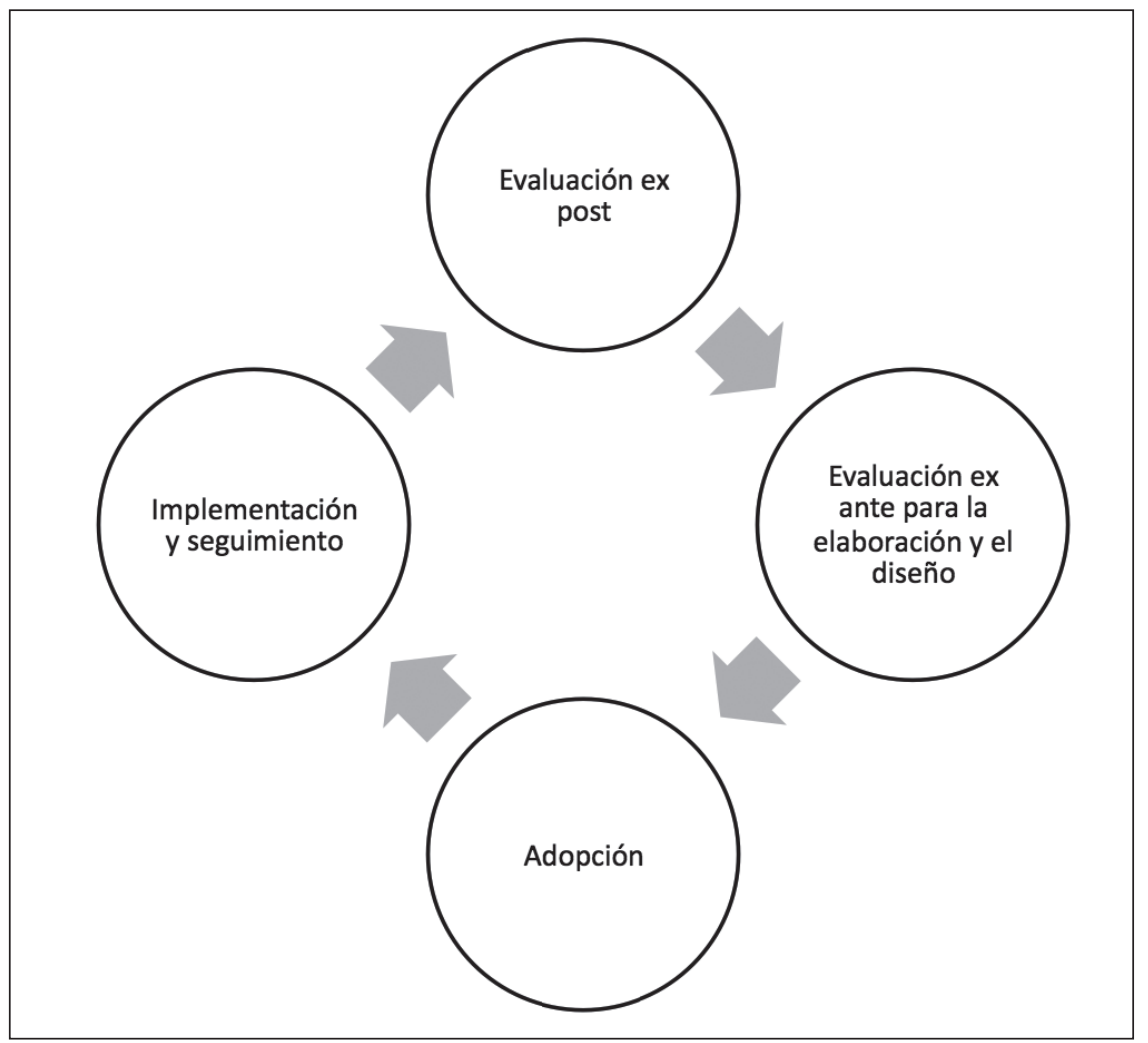

*Fuente: OCDE, "Evaluando Leyes y Regulaciones: El caso de la Cámara de Diputados de Chile" (2012).

Esta doble relación entre la evaluación realizada por diversos órganos institucionales y las diversas etapas del ciclo político-regulatorio, pone en evidencia los importantes beneficios del escrutinio post legislativo, además de ayudarnos a conceptualizar las razones que justifican fomentar el desarrollo del PLS en los parlamentos. En consecuencia, estos motivos pueden ser agrupados en las siguientes tres categorías:

a) Primero, el escrutinio ex post permite mejorar la calidad de la legislación: contribuye a una "mejor regulación" ${ }^{5}$ por diversas vías. Por una parte, el escrutinio post legislativo permite analizar el ciclo político-regulatorio con mayor evidencia, permitiendo identificar cuáles son las falencias de las leyes en vigor y, en consecuencia,

5 El movimiento que buscaba una "mejor regulación" luego y posteriormente de la entrada en vigor de la política intentaba alcanzar "una regulación inteligente", destacando la necesidad de mejorar la calidad de la regulación. Este enfoque ha sido adoptado por la OCDE, la Unión Europea y el Reino Unido. Para más detalles sobre esta agenda, ver WEATHERHILL (2007). 
diseñar los mecanismos que permiten corregir dichas falencias. ${ }^{6} \mathrm{Al}$ mismo tiempo, hace posible identificar las buenas prácticas que podrían ser replicadas en otras leyes. ${ }^{7}$

b) El segundo motivo para involucrar a los parlamentos en la evaluación post legislativa, se relaciona con la idea de mejorar la asignación de responsabilidad a lo largo del proceso legislativo, además de servir como contrapeso al rol que cumple el poder ejecutivo. ${ }^{8}$ En este sentido, el escrutinio post legislativo ayuda a fortalecer el rol representativo y de supervisión de los parlamentos ${ }^{9}$, además de contribuir al desarrollo de sus competencias internas. ${ }^{10}$

c) Por último, existe una razón más general para apoyar el desarrollo del PLS llevado a cabo por los parlamentos, esto es, la posibilidad de mejorar el cumplimiento de las condiciones necesarias para la gobernanza democrática, enfatizando la necesidad de implementar legislación totalmente concordante con los principios de legalidad y seguridad jurídica. ${ }^{11}$ Este punto conecta la evaluación legislativa en general y la evaluación post legislativa en particular, con un estándar más amplio en un Estado de Derecho. ${ }^{12}$

En este artículo me enfocaré en el primer conjunto de beneficios del escrutinio parlamentario post legislativo, debido a que están más relacionados con la racionalidad inmanente al proceso legislativo. La idea de los Parlamentos que promueven el PLS como un "facilitador legislativo", está en línea con la racionalización del proceso de formación de la ley, diseñado para explicar las crecientes complejidades de la legislación en nuestros días. En ese contexto, contar con un sistema institucionalizado de PLS contribuye a la reducción de la ambigüedad y la desconfianza legislativa. ${ }^{13}$

6 Según Hansard, existen fuertes razones para creer que un escrutinio post legislativo más regular y sistemático ayuda a identificar y rectificar problemas en las legislaciones que presentan ciertas deficiencias. Aún más, el saber que las leyes serán monitoreadas constantemente tiene cierto efecto disuasivo, haciendo menos probable promulgar leyes inadecuadas para los respectivos fines. BRAZIER (2005), p. 7.

7 La House of Lords también ha destacado la utilidad del escrutinio post legislativo en el proceso legislativo considerado como un todo. HousE OF LORDS (2004), par. 172.

8 En el proceso legislativo chileno existen varias características que brindan un rol preponderante al poder ejecutivo, incluyendo ciertas materias de iniciativa exclusiva, la posibilidad de acelerar el proceso legislativo, etc. Manzi y otros (2011), p. 25. En el contexto del Parlamento europeo, Zwaan y otros consideran que la evaluación post legislativa apenas ha servido para asignar responsabilidades, pero que se utilizaba más bien con fines prospectivos, con el fin de fijar y modificar la agenda. ZWAAN et al. (2016).

9 BRAZIER (2017).

10 De Vrieze y Hasson (2017), p. 11

11 De VRIEZE y Hasson (2017), p. 11. .

12 Esta conexión ya había sido destacada por la Comisión de Venecia, la que organizó, en junio de 2007, un seminario especializado para servidores públicos de 16 países, quienes se reunieron para compartir experiencias en la materia. Algunas de las ponencias presentadas en el seminario "Evaluación Legislativa" organizado por el campus UNIDE de Trieste, están disponibles en el siguiente enlace: http://www.venice.coe.int/webforms/events/default.aspx?id=650. Último acceso: 12 de febrero de 2018.

13 De Vrieze y Hasson (2017), p. 12. 
Por otro lado, el PLS parlamentario puede presentar ciertos aspectos desventajosos. $\mathrm{Al}$ respecto, se ha dejado en claro que el escrutinio post legislativo no debe ser un escenario que permita revisar los argumentos discutidos durante el debate propio de la aprobación de una ley. Esto significa que el PLS no debe ser una segunda instancia para debatir una ley, sino para examinarla de forma constructiva y orientada hacia el futuro. Asimismo, debe reconocerse que existen costos involucrados en el PLS, en términos de tiempo y recursos, lo que probablemente, se traduciría en la necesidad de priorizar las leyes sujetas al PLS. Finalmente, un enfoque sistemático al PLS requiere de una voluntad política considerable, tanto de parte del gobierno como del parlamento. ${ }^{14}$

En suma, reconocemos que contar con un buen sistema de PLS parlamentario permite verificar si la legislación funciona en la práctica, enfocarse tanto en los objetivos de una determinada política como en su implementación, identificar y difundir buenas prácticas, y, sobre todo, mejorar la regulación. Sin embargo, reconocemos que hay ciertos riesgos aparejados, como que el sistema depende fuertemente de la voluntad política y de los recursos necesarios para llevarlo a cabo. Por lo tanto, un buen sistema de PLS parlamentario tiene que maximizar sus beneficios y a la vez considerar las limitaciones que conlleva. Bajo esta lógica, y con el fin de evaluar qué sistema de PLS es el más idóneo para producir dichos resultados, es necesario especificar cuáles son los criterios comparativos según los cuales haremos un parangón entre los sistemas de PLS de Chile y del Reino Unido.

\section{LOS GRITERIOS COMPARATIVOS}

Los criterios comparativos utilizados como base para este ejercicio se basan en los tres estándares establecidos por Mader: (i) Efectividad, (ii) Eficacia y (iii) Eficiencia. ${ }^{15}$ Según Mader, estos estándares no explican todos los posibles efectos de la legislación, los que designaremos de forma genérica como los "impactos" de la acción legislativa. Sin embargo, permiten enfatizar aspectos especialmente relevantes del proceso de formación de una ley. ${ }^{16}$ A continuación, explicamos en significado de cada uno de estos tres criterios:

i. Efectividad: se refiere a la implementación de la norma. Este estándar exige probar si una norma es o no respetada en la práctica; pero además exige evaluar si la conducta conforme a la norma es o no imputable a esta. ${ }^{17}$

14 De VRieze y Hasson (2017), p. 13.

15 MAder (2001), p. 126.

16 Mader (2011), p. 127.

17 Para los fines de este artículo, ésta será la definición de "efectividad", no obstante, este concepto ha sido debatido y puede ser entendido desde perspectivas diversas (con base en la responsabilidad, la obligatoriedad, la calidad de la legislación, los resultados, etc.) El profesor Stefanou sostiene que falta una teoría general de la efectividad. Así lo señaló en la Conferencia sobre Ley Efectiva y Regulación organizada por el Institute of Advanced Legal Studies, el día 7 de julio de 2017. 
ii. Eficacia: "hasta qué punto la acción legislativa cumple con su objetivo" ["is the extent to which legislative action achieves its goal"']. ${ }^{18}$ Como deja en claro Mader, la implementación (efectividad) es una condición necesaria para la eficacia, pero no es condición suficiente, pues la presunción que subyace a la opción legislativa puede ser incorrecta. Del mismo modo, la eficacia de una norma no es prueba de su efectividad, en cuanto los objetivos de la ley pudieron haber sido alcanzado por factores diversos antes que a causa de norma.

iii. Eficiencia: se enfoca en los costos y beneficios que conlleva una norma. Este análisis va más allá de los beneficios y costos directos financieros o monetarios; también debe tomar en consideración otro tipo de externalidades, incluyendo la carga burocrática, los efectos globales en la economía y hasta los efectos psicológicos producidos en los ciudadanos.

Por cierto, Mader no es el único autor que utilizó estas categorías para definir cuáles debieran ser los criterios de evaluación legislativa. Así, Ulrich Karpen, por ejemplo, también tomó estos mismos estándares como parámetros para evaluar las consecuencias de las leyes. ${ }^{19}$ Desde la perspectiva de la redacción legislativa, Helen Xanthaki se refiere a ellos como las "virtudes" que deben ser alcanzadas. ${ }^{20}$ Otros autores vinculan la calidad de la legislación al cumplimiento de ciertos principios generales del derecho, como la proporcionalidad, la consistencia, la transparencia, la responsabilidad y la eficacia. ${ }^{21}$ Sin embargo, al momento de evaluar el cumplimiento de dichos principios, estos autores reconocen que "a través, en concreto, de la evaluación ex post se promueve la obligación de evaluar periódicamente la aplicación de las normas en vigor [efectividad], con el objeto de comprobar si han cumplido los objetivos perseguidos y si el coste y cargas derivados de ellas estaba justificado y adecuadamente valorado [eficacia]". ${ }^{22}$

La aceptación entre los académicos de estos criterios confirma que pueden ser considerados elementos claves en la calidad de la legislación, y por lo mismo, tiene sentido utilizarlos como una prueba para evaluar un escrutinio parlamentario post legislativo exitoso, en cuanto ellos no se limitan a una perspectiva técnica o procedimental, sino que además controlan el funcionamiento de una ley. Sin embargo, cabe mencionar que ellos no operan como medidas exactas y por eso, sólo deben ser considerados en cuanto guía para evaluar cada modelo de escrutinio post legislativo.

18 Mader (2001), p. 126.

19 Karpen (2004), p. 310.

20 Xanthaki (2010), p. 79.

21 De Montalvo (2017), p. 143.

22 De Montalvo (2017), p. 146. 


\section{COMPARAGIÓN ENTRE LOS MODELOS DE GHILE Y EL REINO UNIDO}

\subsection{El escrutinio post legislativo en Ghile y el Reino Unido}

Antes de evaluar en qué medida cada modelo cumple con los criterios comparativos, es útil describir brevemente el funcionamiento de cada uno de los sistemas de escrutinio, para luego analizar más cuidadosamente los elementos relevantes en términos de efectividad, eficacia y eficiencia.

En el Reino Unido, las referencias a la necesidad e importancia del escrutinio post legislativo se remontan a los 70s. ${ }^{23}$ Sin embargo, desde el año 2004 existen estudios importantes sobre el escrutinio post legislativo; comenzando con el informe del Comité de la House of Lords, ${ }^{24}$ seguido por la respuesta del Gobierno ${ }^{25}$ y el Informe de la Comisión Jurídica. ${ }^{26}$ Después, la oficina del líder de la House of Commons publicó un documento sobre el enfoque gubernamental del escrutinio post legislati$v^{27}$, en respuesta al Informe de la Comisión Jurídica. Desde entonces, la oficina de Gobierno ha incluido constantemente al escrutinio post legislativo en su "Guía de elaboración legislativa" (Guide to Making Legislation). ${ }^{28}$

Actualmente, el sistema de escrutinio post legislativo del Reino Unido funciona de la siguiente forma: (a) de tres a cinco años, luego de la sanción real, el Gobierno presenta (a través del departamento correspondiente) un memorando a los comités de los departamentos que sean relevantes ${ }^{29}$ en la House of Commons ${ }^{30}$ (b) este memorando evalúa la forma cómo la ley ha funcionado en la práctica, considerando los

23 Comisión Jurídica (2006), Par. 2.4.

24 House of Lords (2004).

25 House of Lords (2005).

26 Law Commission (2006).

27 Office of the Leader of the House of Commons (2008).

28 La última versión de esta guía se encuentra en la oficina de gobierno, "Guide to Making Legislation" (2017), parte 43.

29 Tanto la House of Commons como la House of Lords llevan a cabo su trabajo a través de comités, compuestos por entre 10 y 50 miembros del Parlamento o de la House of Lords. Existen diversos tipos de comités, principalmente: (i) Comités Seleccionados que verifican y reportan en áreas que van desde el trabajo de los departamentos gubernamentales, hasta asuntos de índole económica; (ii) Comités Conjuntos compuestos por miembros del Parlamento y de la House of Lords; (iii) Comités Generales, exclusivos de la House of Commons, que examinan en detalle la legislación propuesta, incluyendo a todos los comités antes conocidos como Comisiones Permanentes. Actualmente la House of Commons cuenta con tres Grandes Comités; y (iv) Grandes Comités que tratan las cuestiones relacionadas con Escocia, Gales e Irlanda del Norte.

30 La Comisión Jurídica mencionó el apoyo a los Comités Conjuntos que se someten a un control post legislativo, pero el Gobierno no fue persuadido. En el informe del año 2011, el informe del Leader's Group on Working Practices, de la House of Lords, recomendó establecer un comité permanente de escrutinio post legislativo. Mientras que, en el año 2012, el informe del Comité de Enlace, "Review of select committee activity and proposals for new committee activity" recomendó designar un comité de escrutinio post legislativo ad-hoc para ciertos asuntos. Para ver la evolución de estas propuestas, ver KeLLY y Everett (2013). 
objetivos y criterios de referencia que hayan sido mencionados en la evaluación de impacto, en las notas explicativas o en cualquier otra declaración; (c) luego, el comité decide si realizar o no un escrutinio post legislativo de la ley; (d) el comité puede solicitar un informe completo al departamento respectivo, además de ser posible recibir aportes procedentes de expertos, de la ciudadanía, etc. Se incluye también el apoyo técnico de la Unidad de Escrutinio y de la Oficina Nacional de Auditoría; (e) luego se publica un informe que incluye la evaluación realizada por el comité, con sus conclusiones y recomendaciones finales.

En el Parlamento escocés, el PLS ha sido reconocido explícitamente como parte de las funciones desempeñadas por los comités, y aun cuando a contar del año 2016 existen, específicamente, el Comité para la Auditoría Pública y el Comité de Escrutinio Post Legislativo, se considera que se hallan "otros comités del Parlamento que siempre han podido asumir la labor del PLS y que continuarán haciéndolo" ["other committees of the Parliament have always been able to undertake PLS and will continue to do so" ${ }^{3{ }^{31}}$ Pero lo que es aún más interesante, es que el Parlamento escocés desarrolló una "lista de verificación del escrutinio post legislativo" (Post-legislative scrutiny checklis$t)^{32}$ con el fin de identificar puntos críticos que podrían justificar realizar el PLS. ${ }^{33}$

En Chile, el escrutinio post legislativo se encuentra mucho menos desarrollado. Sin embargo, ha habido esfuerzos para mejorarlo. Así, en el Poder Ejecutivo existen mecanismos de evaluación ex post, vinculados principalmente a la gestión fiscal, incluyendo indicadores de desempeño y programas de evaluación institucional. ${ }^{34}$ Asimismo, también el ejecutivo realiza de manera no sistemática ejercicios de evaluación ex ante. Por otra parte, en el caso del Congreso, los esfuerzos por llevar a cabo un escrutinio post legislativo se han centrado en la Cámara de Diputados. ${ }^{35}$ Tradicionalmente, ha habido talleres temáticos donde se ha realizado, en cierta medida, escrutinio post legislativo, pero éste no es el objetivo principal de los talleres. ${ }^{36}$ Otros autores también mencionan a las Comisiones de Investigación como una forma de escrutinio post legislativo, ${ }^{37}$ pero estas comisiones no persiguen, per se, dicho objetivo.

31 http://www.parliament.scot/parliamentarybusiness/CurrentCommittees/105094.aspx . Último acceso: 16 de febrero de 2018.

32 Disponible en http://www.parliament.scot/parliamentarybusiness/CurrentCommittees/106829. aspx . Último acceso: 16 de febrero de 2018.

33 Dentro de estos puntos críticos, la lista considera, por ejemplo, si la ley lleva en rigor un lapso suficiente como para hacer alguna diferencia, o si la ley puede brindar resultados medibles o los objetivos a alcanzar en una política son medibles, o si la ley cuenta per se con algún mecanismo para el escrutinio post legislativo, etc.

34 OCDE (2012), p. 54

35 En el año 2015 se creó en Chile el Consejo de Modernización para la Labor Legislativa. De forma similar a lo que sucedió en el Reino Unido, este consejo incluyó entre sus propuestas finales, en diciembre del año 2015, la creación de comisiones bicamerales (compuestas por miembros de ambas cámaras), con el fin de tramitar de forma más eficiente los proyectos de ley de fácil despacho. Sin embargo, hasta hoy estas comisiones aún no han sido creadas.

36 OCDE, (2012), p. 56.

37 Paul Díaz y Soto (2009), p. 597. 
Un gran avance se produjo en el año 2011 con la creación del Departamento de Evaluación de la Ley (LED) $)^{38}$ en la Cámara de Diputados, la que cuenta con autonomía funcional con el fin de: (i) evaluar las leyes que han sido aprobadas por el Congreso en cuanto a su eficacia e influencia en la sociedad; (ii) proponer medidas correctivas, de ser necesario, para la correcta aplicación de la ley; y (iii) crear y administrar una red de organizaciones sociales interesadas en participar en la evaluación. ${ }^{39}$

Además, existe un Comité de Evaluación de Ley, integrado por diputados pertenecientes a cada agrupación política, cuya función es cooperar con el trabajo del departamento. Este departamento analiza una determinada ley con miras a resolver si ésta logra cumplir con sus objetivos, identificando los impactos y externalidades, las buenas prácticas, considerando además la percepción de la ciudadanía respecto de ella y proponiendo medidas correctivas. El trabajo del departamento se desarrolla con base en un modelo de tres fases: análisis de ley, percepción de la ciudadanía e informe final. Las leyes evaluadas son seleccionadas cada año por una Mesa de la Cámara de Diputados en base a una lista elaborada por el Departamento de Evaluación de la Ley, siguiendo criterios de neutralidad política, aplicabilidad general, contingencia, factibilidad metodológica, temporal y técnica, además del tiempo de aplicación (al menos un año como ley vigente). ${ }^{40}$ Desde su creación, el departamento ha evaluado diez leyes y una undécima se encuentra actualmente en fase de elaboración del informe.

Existen ciertas características comunes entre los modelos de escrutinio post legislativo de ambos países. En primer lugar, la tarea de realizar el escrutinio es llevada a cabo por la cámara baja en el caso de ambos parlamentos (la House of Commons y la Cámara de Diputados). Sin embargo, en el caso del parlamento de Westminster, se eligió un sistema de control difuso, desarrollado por cada uno de los comités, seleccionados de acuerdo a sus propios criterios; mientras que en Chile, el escrutinio post legislativo es realizado por una unidad centralizada perteneciente a la Cámara de Diputados, la que propone a la mesa de la cámara, las leyes que deben ser evaluadas. Asimismo, ciertos detalles del proceso de evaluación mismo difieren en ambos países, lo que puede impactar en el resultado y en el éxito del escrutinio post legislativo. En la sección siguiente analizaremos dichas características, siempre con base en los criterios identificados.

38 http://www.evaluaciondelaley.cl. Último acceso: 13 de febrero de 2018.

39 Cámara de Diputados (2011).

$40 \operatorname{OCDE}(2012)$, p. 62. 


\subsection{Cumplimiento de los criterios de evaluación}

\subsubsection{Efectividad}

Ambos modelos consideran la efectividad como un criterio clave a ser considerado en el escrutinio post legislativo. Esto explica que en ambos países se considere un período mínimo entre la promulgación de una ley y su PLS. En el caso del Reino Unido, ese período se fijó entre los tres y los cinco años, mientras que en Chile se determinó que sea de al menos un año.

Un aspecto fundamental a considerar, al momento de evaluar la efectividad, es la cooperación con el gobierno o con las agencias encargadas de la implementación, puesto que ellas pueden proveer la mejor información en cuanto al funcionamiento de la ley en la práctica. En el Reino Unido, este elemento está contemplado en la elaboración de un memorando o de un informe completo, que se envía posteriormente al departamento gubernamental respectivo y que es, en definitiva, el punto de partida del escrutinio parlamentario post legislativo. En el caso de Chile, el Departamento de Evaluación de la Ley es el órgano que debe realizar el análisis técnico, incluyendo la opinión de expertos y de las instituciones de implementación. Sin embargo, en Chile, no existe un canal institucional específico para recibir la información, de modo que usualmente sólo es posible acceder a un resumen jurídico de las instituciones involucradas, formalmente, en la aplicación de una ley, mientras que el análisis respecto de la aplicación es efectuado principalmente con base en la percepción ciudadana.

Otro importante aspecto que considerar es si el escrutinio post legislativo cubre o no a la legislación de la cual derivó o aquella que guarda relación con una determinada ley, puesto que "existen muchos detalles y significados contenido en legislación secundaria, además de haber muchas modificaciones de ley o reformas que se efectúan sobre legislación ya existente" ["much of the detail and meaning is contained in the secondary legislation" $y$ "many pieces of legislation amend, or build upon, existing legislation"]. ${ }^{41} \mathrm{En}$ este contexto, tanto en Chile como en el Reino Unido, el escrutinio post legislativo se extiende a la legislación previa, la legislación secundaria, e incluso a la legislación relacionada.

Un método adicional para asegurar la aplicación del escrutinio post legislativo, en términos de efectividad, se relaciona con el uso de cláusulas de caducidad automática. Esta es una técnica legislativa en la cual se incluye, "en una disposición de un proyecto de ley, una fecha de expiración una vez que ésta es promulgada [a provision in a Bill that gives it an expiry date once it is passed into law".${ }^{42}$ Esta técnica puede ser utilizada para mejorar la efectividad de una ley y asegurar la aplicación del escrutinio post legislativo. ${ }^{43}$ Así, es posible invertir la "carga de la prueba", forzando a que quienes

41 BRAZIER (2005), p. 3.

42 http://www.parliament.uk/site-information/glossary/sunset-clause/. Último acceso: 13 de febrero de 2018.

43 Ranchordás (2015), p. 32. 
apoyan una determinada ley prueben su efectividad..$^{44}$ Otros autores destacan que estas cláusulas constituyen una herramienta para reforzar la posición e influencia de las legislaturas y minimizar la influencia del poder ejecutivo en un amplio espectro de los asuntos de política pública. ${ }^{45}$

Las cláusulas de caducidad automática han sido utilizadas de manera creciente en el Reino Unido, en línea con la llamada agenda de "mejor regulación. ${ }^{46}$ No obstante, y aun cuando puede considerarse que estas cláusulas son contrarias al principio de certeza jurídica, no se puede afirmar "que dicho principio se reduzca a la mera estabilidad del Derecho, sino que debe ser considerado como un "concepto multidimensional", que permitirá que el Derecho pueda ser flexible y adaptable". ${ }^{47}$ En esta misma línea, estas revisiones legislativas pueden facilitar la innovación legislativa ${ }^{48}$ o permitir la toma de decisiones basadas en la evaluación del riesgo. ${ }^{49}$

Mientras que en el Reino Unido las cláusulas de caducidad constituyen una herramienta ya explorada e incorporada en el modelo de PLS, en Chile son de muy escasa aplicación. Paul y Soto entregan algunos ejemplos de leyes chilenas que requieren de un período de revisión o de una "fecha de expiración", pero reconocen que se trata de una práctica poco utilizada y limitada usualmente a la revisión de fórmulas matemáticas o de disposiciones técnicas de una ley, destinadas solamente a establecer porcentajes o cifras económicas. ${ }^{50}$

\subsubsection{Eficacia}

Para probar la eficacia es crucial establecer una línea de referencia que permita identificar claramente los objetivos perseguidos por una ley. Estos objetivos pueden estar establecidos en cláusulas de finalidad o en otros documentos, como notas explicativas, memorandos, notas informativas, etc. Las cláusulas de finalidad son disposiciones "que definen los objetivos o metas sociales, económicas o políticas que deben ser alcanzadas" ["that explicitly states the social, economic or political objective or goal that is sought to be achieved']. ${ }^{51}$ A diferencia de las notas explicativas u otros documentos anexos, las cláusulas de finalidad son parte de la ley misma y sirven para clarificar sus objetivos más allá de las interpretaciones de "por qué" la ley fue promulgada, de modo que permiten especificar los objetivos de política y constituir un "baremo" que permita evaluar su eficacia.

\footnotetext{
44 De Montalvo (2017), p. 157.

45 Baugus y Bose (2015), pp. 13-19.

46 RANChORDÁs (2015), p. 33.

47 Ranchordás (2015), p. 45.

48 RANCHORDÁs (2014).

49 Jantz y Veit (2012).

50 Paul Diaz y Soto (2009), pp. 588-560.

51 Berry (2011), p. 49.
} 
En el caso de Chile, los informes del Departamento de Evaluación de la Ley usualmente se refieren a los objetivos ya establecidos en la misma ley, los que rara vez consisten en algo más que un párrafo. En el caso del Reino Unido, además existe una referencia a los propósitos de la ley, con la gran ventaja de que ésta incluye los documentos relativos al escrutinio prelegislativo, como las evaluaciones de impacto, los informes del departamento gubernamental, etc. Desafortunadamente, en Chile no existe un escrutinio prelegislativo sistemático que pueda servir de base para el escrutinio post legislativo, de modo que es importante recordar que aun cuando ambos difieren ${ }^{52}$, serán complementarios. ${ }^{53}$

Otro aspecto importante es quién evalúa el cumplimiento de los objetivos establecidos en una ley. Tal como explicamos antes, en el Reino Unido el escrutinio es llevado a cabo por comités seleccionados, de modo que serán parlamentarios (MPs) quienes evaluarán si una determinada ley alcanzó los objetivos planteados. En el caso de Chile, en cambio, la evaluación es realizada por el Departamento de Evaluación de la Ley, formado por un equipo técnico. En este sentido, no obstante, sostenemos que el escrutinio post legislativo no debe ser una instancia para volver a debatir una ley, sino que consideramos más apropiado que sean los MPs quienes evalúen si ella cumple o no con los objetivos establecidos. Asimismo, si ellos fueron parte de la discusión misma de la ley, estarán mucho más atentos a los detalles y desafíos a los que ésta de origen.

Finalmente, en ambos modelos se considera la participación ciudadana como una forma de asegurar que las personas sometidas a una ley puedan brindar sus apreciaciones respecto de si ella cumple o no con sus objetivos. En el caso de Chile, la metodología elegida por el Departamento de Evaluación de la Ley "otorga especial relevancia a la participación de los individuos y de las agrupaciones sociales sometidos a la ley que se evalúa". ${ }^{54}$ Esto sería importante porque ellos, como afectados directos, "pueden visualizar los efectos colaterales que produce una ley y su participación promueve la transparencia y la responsabilidad del proceso legislativo, además de prevenir la influencia excesiva de intereses particulares por sobre el interés común en el proceso de toma de decisiones" [can help visualize unexpected or unintended side effects of the laws; their participation promotes transparency and accountability to the legislative process; and it prevents the excessive influence of particular interests over the public interest in decision-making $]{ }^{55}$ Siguiendo esta lógica, el Departamento de Evaluación de la Ley administra los foros ciudadanos para la evaluación de la ley, donde individuos y grupos entregan

52 En este sentido, el escrutinio post legislativo no debe ser considerado como una "imagen espejo" del prelegislativo. Law Commission (2006), par. 4.5.

53 Mader (2001), p. 124.

54 http://www.evaluaciondelaley.cl/participacion-ciudadana/foro_ciudadano/2012-11-09/122557. html. Último Acceso: 27 de febrero de 2018.

En la cita original se señala que: "gives special relevance to the participation of individuals and civil society groups related to the law being evaluated"

55 http://www.evaluaciondelaley.cl/participacion-ciudadana/foro_ciudadano/2012-11-09/122557. html . Último Acceso: 27 de febrero de 2018. . 
su opinión, aprehensiones y sugerencias. En el Reino Unido, las opiniones provienen de las ONGs relacionadas con la legislación y de los ciudadanos, quienes son escuchados por los comités como testigos, sin embargo, son considerados como uno más de los agentes relevantes dentro de muchos otros, incluyendo también a expertos, especialistas, académicos, profesionales, etc. ${ }^{56}$

\subsubsection{Eficiencia}

El movimiento internacional para la "mejor regulación" o la "regulación inteligente" $" 57$ se originó desde el inicio de la década del 2000, con especial énfasis en la calidad de la legislación, puesto que ésta era considerada como un instrumento fundamental para mejorar la competitividad y para promover el crecimiento sustentable. Desde entonces, el análisis económico ha sido un elemento crucial de la agenda legislativa en general, y en particular, de la evaluación legislativa. ${ }^{58}$

La prueba de la eficiencia se identifica estrechamente con la evaluación de los costos y beneficios económicos que conlleva una ley y, por lo mismo, normalmente ésta descansa en documentos como las evaluaciones de impacto de la normativa (RIAs) o en los análisis de costo y beneficio $(\mathrm{C} \& \mathrm{~B})$ realizado por el gobierno o por otras agencias en la evaluación ex ante de las políticas.

En el Reino Unido, las RIAs son elaboradas para casi todas las leyes propuestas e incluso se recomienda que incluyan una revisión posterior a la implementación y una descripción de cómo debe ser revisada la política. Este ejercicio permite al Parlamento, posteriormente, efectuar una prueba de la eficiencia del escrutinio post legislativo. Otro factor relevante que permite realizar esta evaluación técnica es el apoyo recibido por parte de los comités especializados, como la Oficina Nacional de Auditoría del Reino Unido y la Unidad de Auditoría. Por ejemplo, la Oficina Nacional de Auditoría informa al Parlamento regularmente sobre las RIAs realizadas por los diferentes departamentos gubernamentales, pudiendo así trazar las áreas a trabajar para mejorar el desempeño, además de producir informes y memorandos que permitan apoyar el trabajo de escrutinio realizado por los comités. ${ }^{59}$

Por otro lado, en Chile "no existe un enfoque metodológico cuantitativo particular para medir los impactos de una ley, por ejemplo, en términos de costo-beneficio" ${ }^{60}$ Aún más, en su descripción metodológica no se mencionan los costos y beneficios de una normativa o la evaluación de su eficiencia. Si bien esta evaluación implica mayores $\operatorname{costos}^{61}$, ya que requiere de la contribución de profesionales altamente capacitados, sería importante incluir un aspecto cuantitativo en un escrutinio post legislativo completo.

56 De VRIEZe (2017), p. 26.

57 Ver nota al pie número 5.

58 Mercado (2013), pp. 95-101.

59 https://www.nao.org.uk/support-for-parliament/ . Último acceso: 27 de febrero de 2018.

60 OCDE (2012), p.63.

$61 \operatorname{Karpen}(2004)$, p. 314. 
En cuanto a los otros tipos de impacto que podrían ser incluidos en la prueba de la eficiencia de una ley, ambos países consideran los impactos más amplios de la misma. El Departamento de Evaluación de la Ley en Chile menciona la necesidad de considerar los aspectos sociales, culturales y ambientales. ${ }^{62}$ Del mismo modo, en el Reino Unido existe una noción amplia del escrutinio post legislativo, que incluye no sólo los efectos jurídicos y económicos de la legislación, sino que también sus consecuencias sociales.

\subsection{Un ejemplo de PLS: Evaluación de la legislación en materia de salud mental}

Para entender mejor el impacto de cada uno de los sistemas de PLS parlamentario, es conveniente revisar un ejemplo de legislación evaluado en ambas jurisdicciones. Ya que ambos parlamentos evaluaron su legislación sobre salud mental, compararé ambos informes para destacar algunas de las diferencias identificadas en las secciones previas.

En marzo del año 2013, el Departamento de Evaluación de la Ley de Chile hizo entrega de un informe referido al Reglamento sobre Discapacidad Mental, la ley $\mathrm{N}^{\circ} 18.600$ del año 1987. ${ }^{63}$ Sólo meses después, en julio del 2013, el Comité de Salud de la House of Commons hizo entrega del PLS sobre la Ley de Salud Mental del año 2007. ${ }^{64}$ Ambos informes parecieran similares debido a su extensión (75 y 87 páginas, respectivamente), sin embargo, el enfoque de su análisis difiere bastante.

La primera diferencia clara se refiere a que, en el caso de Chile, la ley evaluada estaba bastante más desactualizada que la del Reino Unido; de hecho, habían pasado más de veinte años desde su promulgación, lo que probablemente influyó mucho en los resultados del análisis. En cambio, en el caso del Reino Unido, el escrutinio se realizó para una ley reformada sólo seis años antes, lo que permitió considerar sus efectos, pero al mismo tiempo era suficientemente pronto como para identificar posibles fallas y corregirlas hacia el futuro. En este sentido, no obstante ambos sistemas reconocían la necesidad de cierto lapso antes de realizar el PLS, los tres a cinco años establecidos en el Reino Unido resultaron más apropiados en consideración con los ciclos legislativos, mientras que en Chile, el PLS se realizó en algunos casos apenas un año después de la dictación de una ley y en otros veinte o treinta años después, quedando así en evidencia una falta de sistematización en la oportunidad de realizar el PLS, lo que puede debilitar sus impactos.

Analizando el contenido de ambos informes, es posible encontrar ciertas similitudes estructurales. Ambos comienzan contextualizando los antecedentes de la legislación evaluada, incluyendo explícitamente los propósitos y objetivos establecidos en la misma ley. Además, ambos incluyen un análisis de la interacción de la ley en

62 OCDE (2012), p. 62.

63 Departamento de Evaluación de la Ley (2013)

64 Health Committee (2013). 
cuestión con la legislación relacionada; considerando así si se trataba de legislación nacional previa en el ámbito de la Ley sobre Capacidad Mental del Reino Unido de 1983 o del año 2005, si era legislación delegada o si se refería a tratados internacionales. Del mismo modo, la evaluación chilena dedica un capítulo entero a la consistencia de la norma con la Convención Internacional sobre los Derechos de las Personas con Discapacidad.

Sin embargo, el enfoque del análisis no es el mismo. Una de las principales diferencias entre ambos radica en la fuente de la información utilizada en cada caso. En el caso del Reino Unido, la apreciación del Gobierno era la piedra angular de la evaluación realizada por el Comité de Salud. La evidencia reunida para realizar la evaluación de la implementación y ejecución de la ley provenía principalmente de las docenas de informes y de las respuestas del Gobierno enviadas al comité, lo que era contrastado y complementado con la información obtenida gracias a las entrevistas a los profesionales de la salud mental, los usuarios y por la evidencia escrita de las organizaciones del sector y colaboradores anónimos.

En cambio, el informe chileno utilizó principalmente textos jurídicos y académicos para evaluar la legislación, complementado con las encuestas de opinión y los informes de los foros ciudadanos y conferencias, sin embargo, no existía una contribución oficial proveniente de agentes gubernamentales. En este sentido, la única referencia a la implementación era una lista de instituciones públicas que debían lidiar con la ley y de las medidas legislativas adoptadas, pero faltaba una evaluación oficial y datos cuantitativos respecto de cómo estas medidas funcionaban en la práctica.

Respecto de las cuestiones medulares de cada informe, cabe mencionar que el Comité de Salud del Reino Unido fue mucho más detallado y comprehensivo que el chileno. Así, en el primar caso el informe examinó cada medida legislativa adoptada en un capítulo separado (a saber, la prueba sobre tratamiento adecuado, las órdenes y detenciones de la comunidad, los defensores independientes especializados en salud mental, los lugares seguros, el tratamiento comunitario supervisado y las implicancias étnicas de la ley), con revisión explícita de la efectividad, impactos y debilidades de la ley. Asimismo, en el análisis se incluían las presiones financieras y costos involucrados, en línea con el examen de eficiencia. En cambio, en el caso del escrutinio chileno, el informe se enfocaba principalmente en la capacidad jurídica de los individuos con discapacidades mentales, aun cuando esto se debía, en parte, al limitado alcance de la legislación evaluada.

Por último, en cuanto a los resultados del PLS, ambos informes concluían con un valioso capítulo dedicado a entregar recomendaciones precisas. Sin embargo, y debido a la diferencia en el alcance de cada uno de los informes, no resulta sorprendente que las recomendaciones del informe del Reino Unido fueran mucho más integrales que las chilenas.

El informe chileno recomienda eliminar las disposiciones legales relacionadas con la incapacidad de los individuos con discapacidad mental y reemplazarlas por un sistema apropiado, centrado en el apoyo y asistencia, lo que debe ser acorde con los estándares internacionales. Asimismo, el informe destaca que es necesario 
inyectar recursos financieros y humanos para este sistema, además de complementar con acciones de educación y programas de entrenamiento. Otras recomendaciones se relacionaban con algunas reformas propuestas a la figura del representante legal, además de ciertas modificaciones al texto jurídico, refiriéndose a personas con discapacidad mental. Sin embargo, estas recomendaciones no se han traducido en reformas de ley ${ }^{65}$ y aunque en el año 2016 se presentó un proyecto de ley para establecer reglas de protección de los derechos fundamentales de las personas con discapacidad o enfermedades mentales ${ }^{66}$ ante la Cámara de Diputados, éste aún se encuentra en etapa de discusión.

En el caso de las recomendaciones efectuadas en el Reino Unido, el informe del PLS entregó una lista de 25 recomendaciones, incluyendo la recopilación de datos por parte del Departamento de Salud sobre diversas cuestiones que posteriormente deben ser reportadas al Comité, la necesidad de revisar la implementación de algunos de los poderes consagrados en la ley, el alcance de ciertas instituciones importantes, como el defensor de salud mental independiente y el asegurar financiamiento estable para esos servicios, entre otras recomendaciones. Así, aunque no se ha dictado una nueva ley sobre salud mental, cabe mencionar que el Departamento de Salud reconoció estas recomendaciones y dio respuesta a cada una de ellas. ${ }^{67}$ Del mismo modo, el Código de Prácticas sobre Salud Mental resolvió proveer directrices para los profesionales, pacientes, sus familias y cuidadores sobre sus derechos, las que fueron sometidas a un proceso de revisión y actualización en el año $2015 .{ }^{68}$ Además, se reformaron otras guías de implementación sobre la materia. ${ }^{69}$

En consecuencia, este ejemplo específico demuestra que el modelo de PLS parlamentario del Reino Unido está mejor adaptado que el chileno para responder a las pruebas de efectividad, eficacia y eficiencia. Por otro lado, no existe evidencia de que hayan tenido lugar nuevos debates políticos en torno a la legislación evaluada, como consecuencia de la participación de parlamentarios británicos.

65 La única reforma reciente a la Ley $\mathrm{N}^{\circ}$ 18. 600, se refería a la derogación de su artículo 16, en el cual se permitía negociar un contrato de trabajo con una persona con discapacidad mental, estableciendo las partes la remuneración de forma libre, esto es, sin regirse por las normas del ingreso mínimo. Este artículo fue derogado mediante la Ley № 21.015 del año 2017, como parte de la nueva legislación que promueve la inclusión laboral de las personas con discapacidad.

66 Moción $\mathrm{N}^{\mathrm{o}}$ 10755-11, actualmente estudiada por el Senado. Los pasos legislativos pueden ser revisados en el siguiente enlace: https://www.camara.cl/pley/pley_detalle. aspx?prmID=11189\&prmBoletin=10755-11 . Último acceso: 15 de febrero de 2018.

67 La respuesta al informe de PLS está disponible en el siguiente enlace: https://www.gov.uk/ government/uploads/system/uploads/attachment_data/file/252876/33736_Cm_8735_Web_ Accessible.pdf. Último acceso: 15 de febrero de 2018.

68 Más información disponible en el siguiente enlace: https://www.gov.uk/government/publications/ code-of-practice-mental-health-act-1983\#history . Último acceso: 15 de febrero de 2018.

69 Por ejemplo, las guías que implementan cambios a los poderes de la policía y a los lugares para las medidas de seguridad, que fue actualizada en octubre del 2017. La última versión se encuentra disponible en el siguiente enlace: https://www.gov.uk/government/uploads/system/uploads/attachment_data/ file/656025/Guidance_on_Police_Powers.PDF . Último acceso: 15 de febrero de 2018. 


\subsection{Tabla resumen}

\section{Escrutinio Post Legislativo}

\begin{tabular}{|c|c|c|}
\hline $\begin{array}{c}\text { Criterios } \\
\text { Comparativos }\end{array}$ & Reino Unido & Ghile \\
\hline $\begin{array}{l}\text { Efectividad } \\
\text { (implementación) }\end{array}$ & $\begin{array}{l}\text { - Necesidad de esperar entre } \\
\text { tres y cinco años } \\
\text { - Fuertemente basada en in- } \\
\text { formes gubernamentales } \\
\text { - Incluye legislación deriva- } \\
\text { da, previa y relacionada } \\
\text { - Fortalecida por el uso de } \\
\text { cláusulas de caducidad }\end{array}$ & $\begin{array}{l}\text { - Necesidad de esperar al me- } \\
\text { nos un año } \\
\text { - Sin método institucionali- } \\
\text { zado para recibir la retroali- } \\
\text { mentación del Gobierno } \\
\text { - Incluye legislación derivada, } \\
\text { previa y relacionada } \\
\text { - Uso limitado de las cláusulas } \\
\text { de caducidad }\end{array}$ \\
\hline $\begin{array}{l}\text { Eficacia } \\
\text { (objetivos) }\end{array}$ & $\begin{array}{l}\text { - Basada principalmente en } \\
\text { los aportes del escrutinio } \\
\text { prelegislativo } \\
\text { - Evaluada por comités per- } \\
\text { manentes } \\
\text { - Incluye retroalimentación } \\
\text { de la ciudadanía }\end{array}$ & $\begin{array}{l}\text { - Basada en cláusulas de fina- } \\
\text { lidad (sin aporte sistemático } \\
\text { prelegislativo) } \\
\text { · Evaluada por personal téc- } \\
\text { nico (LED) } \\
\text { · Fuerte retroalimentación de } \\
\text { la ciudadanía. }\end{array}$ \\
\hline $\begin{array}{l}\text { Eficiencia } \\
\text { (costos/beneficios) }\end{array}$ & $\begin{array}{l}\text { Uso de la RIA y de anáilis } \\
\text { de costos y beneficios. } \\
\text { Apoyo de unidades espe- } \\
\text { cializadas } \\
\text { Consideración de los im- } \\
\text { pactos en un sentido amplio }\end{array}$ & $\begin{array}{l}\text { - Sin análisis cuantitativo de } \\
\text { costos y beneficios ni apoyo } \\
\text { cuantitativo } \\
\text { - Consideración de los impac- } \\
\text { tos en un sentido amplio }\end{array}$ \\
\hline
\end{tabular}

* Fuente: elaboración propia.

\section{GONGLUSIONES}

Este artículo analizó la figura del escrutinio parlamentario post legislativo. Luego de revisar la relación existente con otros tipos de evaluación, destacamos las ventajas y desventajas del escrutinio post legislativo. Con base en los criterios de Mader, de acuerdo con los cuales una ley es evaluada en cuanto a su efectividad, su eficacia y su eficiencia, desarrollamos un ejercicio comparativo entre los dos modelos de escrutinio parlamentario post legislativo de Chile y del Reino Unido. Éste último se caracteriza por un modelo difuso de evaluación en el cual existen diversos comités permanentes, donde cada uno de ellos tiene la posibilidad de involucrarse en el 
escrutinio post legislativo. En cambio, en el caso chileno se lleva a cabo un modelo de escrutinio post legislativo concentrado, con una unidad especializada al interior de la Cámara de Diputados, la que se encarga del escrutinio post legislativo. En consecuencia, ambos sistemas se diferencian, de manera crucial, en que en el caso del escrutinio post legislativo del Reino Unido éste es efectuado por miembros del Parlamento, mientras que en Chile la tarea la realiza personal técnico parlamentario.

Luego de indagar en los componentes de cada modelo, específicamente la metodología seguida en cada país, confirmamos la hipótesis de que el modelo de escrutinio post legislativo parlamentario del Reino Unido cumple mejor los criterios de Mader, esto debido a que aborda cada uno de los estándares de forma mucho más integral que en el modelo chileno. El caso de estudio de escrutinio post legislativo, sobre la legislación de salud mental y que fue desarrollado por ambos Parlamentos, confirmaron nuestro hallazgo.

En este contexto, es posible distinguir tres tipos de recomendaciones para el modelo de escrutinio parlamentario post legislativo chileno: (i) coordinar e institucionalizar un método para que exista cooperación con el gobierno, con el fin de evaluar mejor la implementación de una ley (efectividad); (ii) mejorar y regularizar el ejercicio del escrutinio ex ante, para así generar insumos útiles que permitan determinar si una ley alcanza sus objetivos (eficacia); y (iii) reforzar el Departamento de Evaluación de la Ley, asegurando que incorpore un enfoque cuantitativo, que permita el análisis de la legislación en cuanto a sus costos y beneficios (eficiencia). ${ }^{70}$ Sin embargo, es seguro que realizar estas modificaciones requerirá de apoyo político y de recursos.

En el caso del Reino Unido, el parlamento de Westminster desarrolló un completo proceso de escrutinio post legislativo, con una estrecha cooperación con el gobierno y el parlamento. Sin embargo, este sistema puede tener un enfoque más sistemático en términos de definir, claramente, los criterios para seleccionar las leyes que serán sujetas al escrutinio. Eso sí, manteniendo la flexibilidad necesaria para adaptarse a las circunstancias cambiantes. Debido a las limitaciones en cuanto a tiempo y recursos, el parlamento no puede realizar el escrutinio de todas las leyes y se ve obligado a priorizar, pero sería importante que los criterios de priorización fueran claros y transparentes. Un buen ejemplo a seguir, sería la lista de verificación del escrutinio del Parlamento escocés, puesto que existen algunos puntos que dan lugar al escrutinio post legislativo, lo que contribuiría a institucionalizar este sistema de evaluación.

Por último, una recomendación válida para Chile y para el Reino Unido en cuanto al escrutinio parlamentario post legislativo, tiene relación con la necesidad de desarrollar un marco común para cada una de sus cámaras. A lo largo de este artículo hemos confirmado que el escrutinio post legislativo parlamentario es llevado a cabo principalmente por la House of Commons de Westminster y por la Cámara

70 Estas recomendaciones son consistentes con aquellas efectuadas por la OCDE en su informe del año 2012, donde se distinguió entre las recomendaciones institucionales, metodológicas y de gobernanza. OCDE (2012), p. 75. 
de Diputados, sin embargo, esto no implica que la House of Lords o que el Senado, respectivamente, puedan realizar el escrutinio post legislativo. Esto conlleva un riesgo de estar duplicando funciones o incluso, de arribar a conclusiones contradictorias. La creación de este marco común no debe traducirse necesariamente en comisiones conjuntas o en un departamento de evaluación del common law, pero al menos, debería establecer canales formales de comunicación, para así evitar la duplicación y promover las sinergias entre ambas cámaras. 


\section{BIBLIOGRAFÍA CITADA}

Baugus, Brian y Bose, Feler (2015). Sunset Legislation in the States: Balancing the Legislature and the Executive (Mercatus Center, George Mason University).

Berry, Duncan (2011). "Purpose sections: Why they are a good idea for drafters and users", Loophole, Mayo, pp. 49-67.

Brazier, Alex (2005). "Post-Legislative Scrutiny", en Briefing Paper: Issues in Law Making, Vol. 6, Hansard Society.

Brazier, Alex (2017). "Post legislative scrutiny”, en Guide to Parliaments, Vol. 8, Global Partners Governance.

Cabinet Office (2017): “Guide to Making Legislation”, en <https://www.gov.uk/ government/uploads/system/uploads/attachment_data/file/616341/Guide_to_Making_Legislation_Apr_2017_v2.0_final_May_update.pdf> último acceso el 13 de febrero de 2018.

De Montalvo, Federico (2017). "La evaluación ex post de las normas: un análisis del nuevo modelo español", Revista Parlamentaria de la Asamblea de Madrid, № 36, pp. 139-177.

De VRIeze, Franklin y Hasson, Victoria (2017). Post legislative scrutiny. Comparative study of practices of Post legislative scrutiny in selected parliaments and the rationale for its place in democracy assistance (Westminster Foundation for Democracy).

De VRIEze, Franklin (2017). Post-Legislative Scrutiny: guide for parliaments (Westminster Foundation for Democracy).

Department of Business Innovation and Skills [BIS] (2010). Clarifying the Relationship between Policy Evaluation, Post legislative scrutiny and Post-Implementation Review.

Departamento de Evaluación de la Ley de la Cámara de Diputados (2013). Evaluación de la Ley $\mathcal{N}^{\circ} 18.600$ que Establece normas sobre discapacitados mentales.

House of Commons, Health Committee (2013). Post legislative scrutiny of the Mental Health Act 2007.

House of Lords, Select Committee on the Constitution (2004). Parliament and the Legislative Process, $14^{\text {th }}$ Report, Session 2003-04, HL Paper 173-I.

House of Lords, Select Committee on the Constitution (2005). Parliament and the Legislative Process: The Government's Response, $6^{\text {th }}$ Report, Session 2004-05, HL Paper 114.

Jantz, Bastian y VeIt, Sylvia (2012). "Sunset Legislation: Theoretical Reflections and International Experiences", en Alemanno A., Den Butter F., Nijsen A. y TORriti J. (eds.), Better Business Regulation in a Risk Society (Springer), pp. 267-282. 
KARPen, Ulrich (2004). "La evaluación de las consecuencias de las leyes", Anuario Iberoamericano de Fusticia Constitucional, No. 8, pp. 307-316.

Kelly, Richard y Everett, Michael (2013). Post legislative scrutiny, House of Commons, Parliament and Constitution Centre.

Law Commission (2006). Post legislative scrutiny. A Consultation Paper, Consultation Paper $\mathrm{N}^{\circ} 178$.

Mader, Luzius (2001). "Evaluating the Effects: A Contribution to the Quality of Legislation", Statute Law Review, Vol. 22, № 2, pp. 119-131.

Manzi, Jorge; Cortés, Flavio, Mardones, Rodrigo, Riveros, Alejandra y Toro, Sergio (2011). "Un enfoque de gestión para mejorar la eficacia legislativa en Chile", en Concurso Políticas Públicas: Propuestas para Chile (Centro de Políticas Públicas UG), pp. 23-60.

Mercado, Pedro (2013). "Calidad de la Ley, evaluación de impacto normativo y argumentos económicos”, Anales de la Cátedra Francisco Suárez, No 47, pp. 85-108.

OECD (2012): Evaluating Laws and Regulations: The Case of the Chilean Chamber of Deputies (OECD Publishing). DOI: <http://dx.doi.org/10.1787/9789264176263-en>

Office of the Leader of the House of Commons (2008). Post legislative scrutiny - The Government's Approach.

Paul Diaz, Álvaro y Sото Sebastián (2009). "Legislar con eficiencia: la importancia de evaluar las leyes", Revista Actualidad furídica, N 19, pp. 585-614.

Ranchordás, Sofia (2014). Sunset clauses and experimental legislation: Blessing or curse for innovation (Koninklijke Wöhrmann B.V.).

Ranchordás, Sofia (2015). "Sunset Clauses and Experimental Regulations: Blessing or Curse for Legal Certainty”, Statute Law Review, Vol. 36, Issue 1, pp. 28-45.

Stefanou, Constantin (2017). The policy and economics of legislative and regulatory effectiveness Panel, Conference on Effective Law and Regulation, Institute of Advanced Legal Studies.

Weatherill, Stephen (ed.) (2007). Better Regulation (Hart Publishing).

XANTHAKi, Helen (2010). "Quality of legislation: an achievable universal concept or a utopian pursuit?", en Mader, Luzius y Tavares de Almeida, Marta (eds.), Quality of Legislation. Principles and Instruments: Proceedings of the Ninth Congress of the International Association of Legislation (IAL) (Nomos), pp. 75-86.

Zwaan, Pieter, Van Voorst, Stijn y Mastenbroek, Ellen (2016). "Ex post legislative evaluation in the European Union: questioning the usage of evaluations as instruments for accountability", International Review of Administrative Sciences (IRAS), Vol. 82, Issue 4, pp. 674-693. 


\section{NORMAS GITADAS}

\section{Ghile:}

Ley $\mathrm{N}^{\circ} 18.600$ del 1 de abril de 2018, que establece normas sobre deficientes mentales.

Ley $N^{\circ} 21.015$ del 15 de junio de 2017, que incentiva la inclusión de personas con discapacidad al mundo laboral. 
